\title{
Designated Blood Product Donation
}

National Cancer Institute

\section{Source}

National Cancer Institute. Designated Blood Product Donation. NCI Thesaurus. Code C133270.

Donations from a donor called by the collecting facility to provide products for a specific recipient in some future therapeutic procedure, e.g. specifically matched blood for patients with antibodies. 